\title{
Van der Waals Attraction
}

National Cancer Institute

\section{Source}

National Cancer Institute. van der Waals Attraction. NCI Thesaurus. Code C73474.

The weak attraction between two or more chemical entities that is caused by the polarization of adjacent atoms or molecules. 